\title{
Simultaneous and Systemic Knock-down of Big Defensin 1 and 2 gene Expression in the Pacific Oyster Crassostrea gigas using Long Double-stranded RNA-mediated RNA Interference
}

\author{
Bo Young Jee ${ }^{1 \dagger}$, Min Sun Kim ${ }^{3 \dagger}$, Mi Young Cho ${ }^{1}$, Soon Jeong Lee ${ }^{1}$, Myung Ae Park ${ }^{1}$, Jin Woo Kim², \\ Seung Hyuk Choi ${ }^{3}$, Hyun Do Jeong ${ }^{3}$ and Ki Hong Kim ${ }^{3 *}$ \\ ${ }^{1}$ Aquatic Life Disease Control Division, National Fisheries Research and Development Institute, Busan 619-902, Korea \\ ${ }^{2}$ Pathology division, National Fisheries Research and Development Institute, Busan 619-902, Korea \\ ${ }^{3}$ Department of Aquatic Life Medicine, Pukyong National University, Busan 608-737, Korea
}

\begin{abstract}
RNA interference (RNAi)-mediated transcriptional knock-down of Crassostrea gigas big defensin 1 and 2 genes (Cg-BigDef1 and $C g$-BigDef2) was investigated. The cDNA sequences of $C g$-BigDef1 and $C g$-BigDef2 were identical, excluding an additional fragment of 20 nucleotides in $C g$-BigDefl; thus, a long double-stranded RNA (dsRNA) targeting the mRNA of $C g$-BigDef2 effectively downregulated both $C g$-BigDef2 and $C g$-BigDef1. In addition, long dsRNA targeting green fluorescent protein (GFP) did not affect transcription of the two big defensin genes. These results suggest that the transcriptional downregulation of $\mathrm{Cg}$-BigDef1 and $\mathrm{Cg}$-BigDef2 was mediated by sequence-specific RNA interference (RNAi). Despite injection of long dsRNA targeting $\mathrm{Cg}_{-}$ BigDef2 into only the adductor muscle, knock-down of $C g$-BigDef1 and $C g$-BigDef2 was observed in the adductor muscle, hemocytes, mantle, and gills, suggestive of systemic spread of RNAi in C. gigas. Furthermore, the inhibitory effect of dsRNA persisted until $72 \mathrm{~h}$ post-injection, indicative of a long-lasting RNAi-mediated knock-down of target genes.
\end{abstract}

Key words: Crassostrea gigas, Long double-stranded RNA, RNA interference, Big defensins

\section{Introduction}

RNA interference (RNAi), a highly conserved cellular mechanism in eukaryotic organisms, is a form of post-transcriptional homologous gene-silencing induced by doublestranded RNA (dsRNA) (Fire et al., 1998; Fire, 1999). DsRNA in cells is cleaved into small dsRNA fragments called the short interfering RNA (siRNA) by an RNase III-like enzyme, Dicer. These fragments are then incorporated into the RNA-induced silencing complex (RISC), which can degrade target mRNA in a sequence-specific manner, resulting in knock-down of the expression of the target gene (Bernstein et al., 2001; Sharp, 2001; Zamore, 2001; Hannon, 2002). Although RNAi-medi- ated knockdown of specific genes has been demonstrated in some species of gastropods, such as Aplysia (Lee et al., 2001), Lymnaea stagnalis (Korneev et al., 2002), and Biomphalaria glabrata (Jiang et al., 2006), and in bivalves such as the Pacific oyster Crassostrea gigas (Fabioux et al., 2009; Huvet et al., 2012) and Zhikong scallop Chlamys farreri (Wang et al., 2011), RNAi has not commonly been applied in mollusks.

Antimicrobial peptides (AMPs) are small amphipathic peptides that play a role in the innate defense of hosts against microbial infections (Hancock and Scott, 2000; Bulet et al., 2004; Otero-González et al., 2010). Big defensin, initially
(C) 2014 The Korean Society of Fisheries and Aquatic Science This is an Open Access article distributed under the terms of the Creative Commons Attribution Non-Commercial Licens (http://creativecommons. org/licenses/by-nc/3.0/) which permits unrestricted non-commercial use, distribution, and reproduction in any medium, provided the original work is properly cited.
Received 28 June 2013; Revised 24 March 2014

Accepted 16 April 2014

*Corresponding Author

E-mail: khkim@pknu.ac.kr

${ }^{\dagger}$ Both authors contributed equally to this work. 
identified in the horseshoe crab Tachypleus tridentatus (Saito et al., 1995), is a type of AMP with two structurally and functionally different domains. The N-terminal domain is highly hydrophobic and more active against Gram-positive bacteria, while the C-terminal domain is cysteine-rich, cationic, and more active against Gram-negative bacteria (Saito et al., 1995). In bivalve mollusks, several big defensins have been reported. Zhao et al. (2007) identified the first one from bay scallop (Argopecten irradians) and showed that it had microbicidal activity against Gram-positive and Gram-negative bacteria and fungi. In the hard clam (Mercenaria mercenaria), expression of a big defensin gene significantly increased after challenge with a protistan parasite called quahog parasite unknown (Perrigault et al., 2009). A big defensin identified from clam (Venerupis philippinarum) showed increased transcription after Vibrio anguillarum challenge, and exhibited bactericidal activity against both Gram-positive and Gram-negative bacteria (Zhao et al., 2010). Recently, Gerdol et al. (2012) reported eight big defensins from the Mediterranean mussel (Mytilus galloprovincialis). In the Pacific oyster (Crassostrea gigas), six AMPs including three defensins ( $C g$-Def, $C g$-Defh1, $C g$-Defh2) and three big defensins ( $C g$-BigDef1, $C g$-BigDef2, CgBigDef3) have been reported (Gueguen et al., 2006; Gonzalez et al., 2007; Rosa et al., 2011). Among the three big defensins, $C g$-BigDef1 and $C g$-BigDef2 were transcriptionally upregulated after injection with heat-killed or live bacteria (Rosa et al., 2011).

Although various methods can be used to analyze gene function, RNAi-mediated knock-down of gene expression is a convenient method for in vivo analysis of gene function. However, for in vivo experiments, it is important to understand whether the RNAi effect is systemic and how long the RNAi effect will last. In the present study, we investigated whether focal injection of a long dsRNA targeting $C g$-BigDef 2 could systemically induce RNAi-mediated transcriptional knockdown of $C$. gigas big defensin 1 and 2 genes over time.

\section{Materials and Methods}

\section{Long double-stranded RNA (dsRNA) preparation}

Double-stranded RNAs (dsRNAs) specific to oyster big defensin 2 ( $C g$-BigDef2) and to green fluorescent protein (GFP) were synthesized in vitro using the Megascript RNAi kit (Ambion, Austin, TX, USA) according to the manufacturer's instructions. Briefly, DNA fragments partially encoding cDNA of $\mathrm{Cg}$-BigDef2 and GFP genes were amplified via polymerase chain reaction (PCR) with gene-specific primers (Table 1) and cloned into the pGEM T-easy vector (Promega, Madison, WI, USA). After confirming the sequences, plasmids containing $\mathrm{Cg}$-BigDef2 and GFP were digested with EcoRI/NcoI and EcoRI/HindIII, respectively. Then these genes were subcloned into the LITMUS 28i vector (New England Biolabs, Ipswich,
MA, USA) and named pdT7-BigDef2 and pdT7-GFP, respectively. For single-stranded RNA (ssRNA) production, each vector was linearized with EcoRI and in vitro transcription was performed. Each antisense strand was synthesized after linearizing the vector with $\mathrm{NcoI}(\mathrm{Cg}$-BigDef2) or HindIII (GFP). Equal amounts of sense and antisense RNA were annealed to produce dsRNA. Digestion of the template DNA and any ssRNA remaining in the dsRNA, as well as purification of dsRNA, were performed using reagents in the Megascript kit (Ambion).

\section{Oysters and dsRNA administration}

Pacific oysters (C. gigas; shell length 6-8 cm) were obtained from a commercial oyster farm in Korea and maintained in filtered seawater at $20^{\circ} \mathrm{C}$. For the experiment, 36 oysters were randomly selected, divided into 3 groups of 12 oysters, put into a 250 -L tank, and acclimated for 1 week without changing the water. After starting the injection experiment, cultured water in the tank was completely replaced once a day with newly filtered seawater. Oysters in each group were injected with dsRNA targeting $C g$-BigDef2 or GFP into the adductor muscle at a dose of $50 \mu \mathrm{g}$ per oyster. Oysters in the control group were injected with phosphate buffered saline (PBS) alone. At 12, 24, 48 and $72 \mathrm{~h}$ post-injection, three oysters in each group were randomly removed to analyze knock-down of target gene expression.

\section{Effect of long dsRNAs on the expression of $\mathrm{Cg}$ - BigDef1 and two genes in C. gigas}

Hemolymph withdrawn from the adductor muscle was immediately centrifuged at $1,000 \mathrm{~g}$ for $20 \mathrm{~min}$ at $4^{\circ} \mathrm{C}$ to obtain hemocytes. A part of the adductor muscle, as well as the mantle and gill, were excised from each oyster. Total RNA was extracted using RNAiso plus reagent (Takara, Shiga, Japan). Prior to synthesizing first-strand cDNA, $1 \mu \mathrm{g}$ total RNA was treated with RNase-free DNaseI (Promega). To synthesize first-strand cDNA, $1 \mu \mathrm{g}$ total RNA treated with DNase was incubated with $0.5 \mu \mathrm{L}$ random primer $(0.5 \mu \mathrm{g} / \mathrm{mL}$, Promega) at $80^{\circ} \mathrm{C}$ for $5 \mathrm{~min}$ and further incubated at $42^{\circ} \mathrm{C}$ for $60 \mathrm{~min}$ in reaction mixture containing $2 \mu \mathrm{L}$ each of $10 \mathrm{mM}$ dNTP mix (Takara), $0.5 \mu \mathrm{L}$ M-MLV reverse transcriptase (Promega), and $0.25 \mu \mathrm{L}$ RNase inhibitor (Promega) in a final reaction volume of $10 \mu \mathrm{L}$. PCR was performed with $2 \times$ Prime Taq Premix (Genet Bio, Daejeon, Korea), $1 \mu \mathrm{L} 10^{-1}$ diluted cDNA template, and oligonucleotide primer pairs for amplification of the each target BigDef gene ( $C g$-BigDef $1,2-\mathrm{F}$ and $C g$-BigDef 1-R or $C g$-BigDef 2-R) and the control elongation factor (EF) gene (EF-F and EF-R) (Table 1). Thermal cycling conditions were 1 cycle of $3 \mathrm{~min}$ at $95^{\circ} \mathrm{C}$ (initial denaturation) followed by 35 cycles (for $C g$-BigDef1), 27 cycles (for $C g$-BigDef2), or 25 cycles (for $\mathrm{EF}$ ) of $30 \mathrm{~s}$ at $95^{\circ} \mathrm{C}, 30 \mathrm{~s}$ at $60^{\circ} \mathrm{C}$, and $30 \mathrm{~s}$ at $72^{\circ} \mathrm{C}$, with a final extension step of $7 \mathrm{~min}$ at $72^{\circ} \mathrm{C}$. PCR 

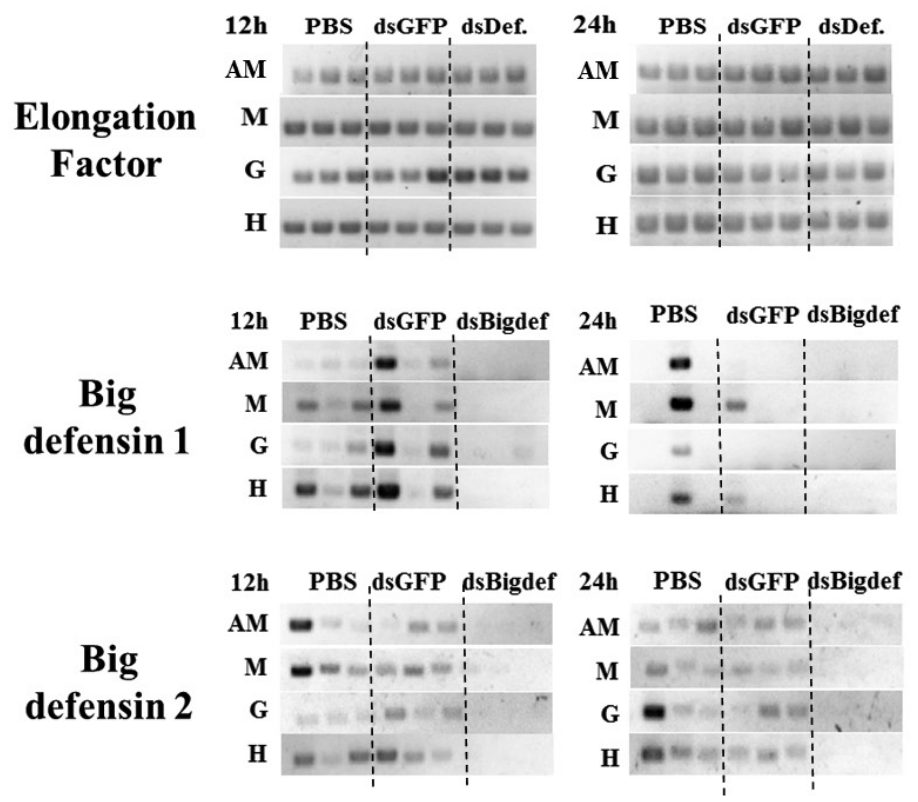
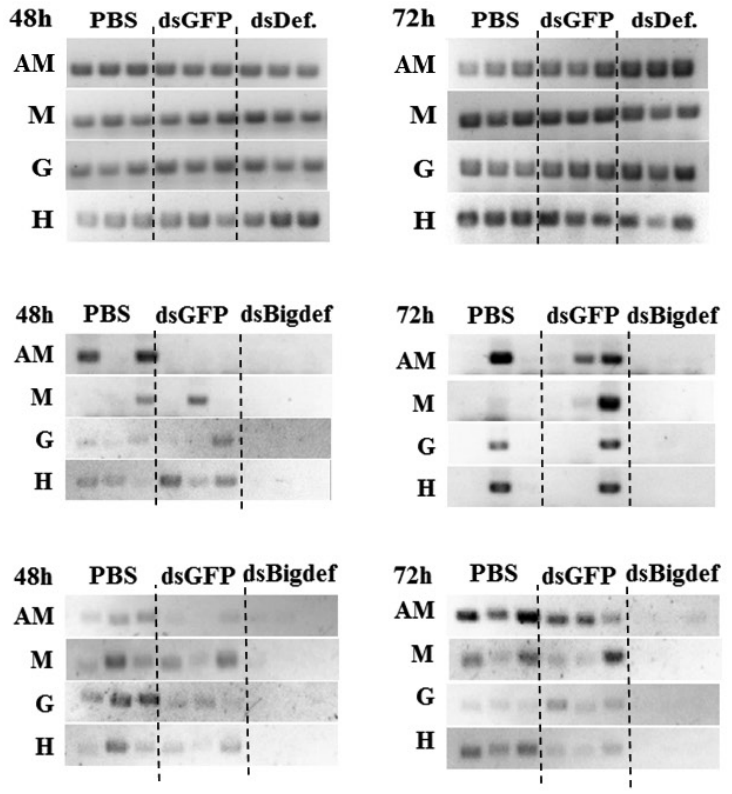

Fig. 1. RNA interference of big defensin 1 and 2 expression in oysters Crassostrea gigas by long double-stranded RNA (dsRNA). Transcriptional expression of elongation factor (control), big defensin 1 and big defensin 2 genes in adductor muscle (AM ), mantle (M), gill (G), and hemocytes (H) in oysters Crassostrea gigas that were injected with long dsRNA targeting mRNA of big defensin 2 (dsDef), green fluorescent protein (dsGFP) or phosphate buffered saline (PBS) into adductor muscle. At each 12, 24, 48 and $72 \mathrm{~h}$ post-injection, 3 oysters in each group were randomly sampled and transcription of the genes was analyzed by RT-PCR. Bands represent transcripts of each individual oyster $(n=3)$ in each group.

samples to be compared were electrophoresed on $1 \%$ agarose gels and stained with ethidium bromide $(\mathrm{EtBr})$.

\section{Results}

Transcription of the $\mathrm{Cg}$-BigDef2 gene was clearly downregulated by injection of long dsRNA targeting the $\mathrm{Cg}$-BigDef2 gene (Fig. 1). Transcription of the $C g$-BigDefl gene also decreased after injection of long dsRNA targeting the $\mathrm{Cg}$-BigDef2 gene. However, expression of the $C g$-BigDef2 gene in oysters was not affected by injection of long dsRNA targeting the GFP gene.

Injection of long dsRNA targeting mRNA of the $\mathrm{Cg}$-BigDef2 gene into the adductor muscle of oyster induced knockdown of the two BigDef genes in that muscle, as well as in the mantle, gill, and hemocytes (Fig. 1). Transcriptional knockdown of $C g$-BigDef1 and $C g$-BigDef2 genes in oysters injected with long dsRNA targeting the $\mathrm{Cg}$-BigDef2 gene persisted until $72 \mathrm{~h}$ post-injection (Fig. 1).

\section{Discussion}

Long dsRNA targeting mRNA of $C g$-BigDef2 transcriptionally downregulated both $C g$-BigDef1 and $C g$-BigDef2. Because cDNA sequences of $C g$-BigDef1 and $C g$-BigDef2 are identical excluding an additional fragment of 20 nucleo- tides in $C g$-BigDefl (Rosa et al., 2011), transcription of both $C g$-BigDef1 and $C g$-BigDef2 may be suppressed by siRNAs originating from long dsRNA targeting $\mathrm{Cg}$-BigDef2. Furthermore, we did not observe a decrease in expression of the two big defensin genes after injection of long dsRNA targeting GFP, suggesting that the knock-down of $C g$-BigDefl and $C g$ BigDef2 was mediated by a sequence-specific RNAi phenomenon. Although Rosa et al. (2011) reported that hemocytes are the only tissues that express $C g$-BigDefs, we found mRNAs of $C g$-BigDefl and 2 in hemocytes as well as other tissues, including the adductor muscle, mantle, and gill, which may be the result of hemocyte infiltration. Rosa et al. (2011) also indicated that other cell types can express $C g$-BigDef genes.

In some organisms including Drosophila and vertebrates, dsRNA taken up by a cell is not transmitted to other cells (Roignant et al., 2003; Bitko et al., 2005), whereas in cells of Caenorhabditis elegans and some other invertebrates, dsRNA can spread to other cells, resulting in a systemic RNAi response (Winston et al., 2002; Dong and Friedrich, 2005). Fabioux et al. (2009) and Huvet et al. (2012) reported dsRNAmediated knock-down of target genes in oysters. However, the injection site and expression analysis in their experiments were restricted to the gonad, and little information is available on the use of systemic RNAi in oysters. In the present study, despite the injection of BD2-dsRNA into only the adductor muscle, knock-down of $\mathrm{Cg}$-BigDef1 and 2 was observed not only in the muscle but also in hemocytes, the mantle, and gills, suggestive of systemic spread of RNAi in C. gigas. 
The antimicrobial activity of molluscan big defensins has been demonstrated by analyzing in vitro bactericidal activity using recombinant big defensins or shown indirectly based on transcriptional upregulation of big defensin genes in response to bacterial infection (Zhao et al., 2007, 2010; Perrigault et al., 2009; Rosa et al., 2011). In the present study, we systemically downregulated two big defensin genes in $C$. gigas using RNAi, which can be used in future studies to analyze the functions of big defensins in oyster.

\section{Acknowledgments}

This research was supported by the National Fisheries Research \& Development Institute (NFRDI) (RP-2013-AQ-106).

\section{References}

Bernstein E, Denli AM and Hannon GJ. 2001. The rest is silence. RNA 7, 1509-1521.

Bitko V, Musiyenko A, Shulyayeva O and Barik S. 2005. Inhibition of respiratory viruses by nasally administered siRNA. Nat Med 11, 50-55.

Bulet P, Stocklin R and Menin L. 2004. Anti-microbial peptides: from invertebrates to vertebrates. Immunol Rev 198, 169-184.

Dong Y and Friedrich M. 2005. Nymphal RNAi: systemic RNAi mediated gene knockdown in juvenile grasshopper. BMC Biotechnol 5,25 .

Fabioux C, Corporeau C, Quillien V, Favrel P and Huvet A. 2009. In vivo RNA interference in oyster - vasa silencing inhibits germ cell development. FEBS J 276, 2566-2573.

Fire A. 1999. RNA-triggered gene silencing. Trends Genet 15, 358-363.

Fire A, Xu S, Montgomery MK, Kostas SA, Driver SE and Mello CC. 1998. Potent and specific genetic interference by double-stranded RNA in Caenorhabditis elegans. Nature 391, 806-811.

Gerdol M, De Moro G, Manfrin C, Venier P and Pallavicini A. 2012. Big defensins and mytimacins, new AMP families of the Mediterranean mussel Mytilus galloprovincialis. Dev Comp Immunol 36, 390-399.

Gonzalez M, Gueguen Y, Desserre G, de Lorgeril J, Romestand B and Bachère E. 2007. Molecular characterization of two isoforms of defensin from hemocytes of the oyster Crassostrea gigas. Dev Comp Immunol 31, 332-339.

Gueguen Y, Herpin A, Aumelas A, Garnier J, Fievet J, Escoubas JM, Bulet P, Gonzalez M, Lelong C, Favrel P. and Bachère E. 2006. Characterization of a defensin from the oyster Crassostrea gigas. Recombinant production, folding, solution structure, antimicrobial activities, and gene expression. J Biol Chem 281, 313-323.

Hancock REW and Scott MG. 2000. The role of antimicrobial peptides in animal defenses. Proc Nat'l Acad Sci USA 97, 8856-8861.

Hannon GJ. 2002. RNA interference. Nature 418, 244-251.

Huvet A, Fleury E, Corporeau C, Quillien V, Daniel JY, Riviere G,
Boudry P and Fabioux C. 2012. In vivo RNA interference of a gonad-specific transforming growth factor- $\beta$ in the Pacific oyster Crassostrea gigas. Mar Biotechnol 14, 402-410.

Jiang Y, Loker ES and Zhang SM. 2006. In vivo and in vitro knockdown of FREP2 gene expression in the snail Biomphalaria glabrata using RNA interference. Dev Comp Immunol 30, 855-866.

Korneev SA, Kemenes I, Straub V, Staras K, Korneeva EI, Kemenes G, Benjamin PR and O'Shea M. 2002. Suppression of nitric oxide (NO)-dependent behavior by double-stranded RNA mediated silencing of a neuronal NO synthase gene. J Neurosci 22, RC227, $1-5$.

Lee JA, Kim HK, Kim KH, Han JH, Lee YS, Lim CS, Chang DJ, Kubo $\mathrm{T}$ and Kaang BK. 2001. Overexpression of and RNA interference with the CCAAT enhancer-binding protein on long-term facilitation of Aplysia sensory to motor synapses. Learn Mem 8, 220-226.

Otero-González AJ, Magalhães BS, Garcia-Villarino M, López-Abarrategui C, Sousa DA, Dias SC and Franco OL. 2010. Antimicrobial peptides from marine invertebrates as a new frontier for microbial infection control. FASEB J 24, 1320-1334.

Perrigault M, Tanguy A and Allam B. 2009. Identification and expression of differentially expressed genes in the hard clam, Mercenaria mercenaria, in response to quahog parasite unknown (QPX). BMC Genomics 10, 377-394.

Roignant JY, Carré C, Mugat B, Szymczak D, Lepesant JA and Antoniewski C. 2003. Absence of transitive and systemic pathways allows cell-specific and isoform specific RNAi in Drosophila. RNA 9, 299-308.

Rosa RD, Santini A, Fievet J, Bulet P, Destoumieux-Garzón D and Bachère E. 2011. Big defensins, a diverse family of antimicrobial peptides that follows different patterns of expression in hemocytes of the oyster Crassostrea gigas. PLoS ONE 6, e25594.

Saito T, Kawabata S, Shigenaga T, Takayenoki Y, Cho J, Nakajima H, Hirata M and Iwanaga S. 1995. A novel big defensin identified in horseshoe crab hemocytes: Isolation, amino acid sequence, and antibacterial activity. J Biochem 117, 1131-1137.

Sharp PA. 2001. RNA interference-2001. Genes Develop 15, 485-490.

Wang M, Yang J, Zhou Z, Qiu L, Wang L, Zhang H, Gao Y, Wang X, Zhang L, Zhao J and Song L. 2011. A primitive Toll-like receptor signaling pathway in mollusk Zhikong scallop Chlamys farreri. Dev Comp Immunol 35, 511-520.

Winston WM, Molodowitch C and Hunter CP. 2002. Systemic RNAi in C. elegans requires the putative transmembrane protein SID-1. Science 295, 2456-2459.

Zamore PD. 2001. RNA interference: listening to the sound of silence. Nat Struct Biol 8, 746-750.

Zhao J, Song L, Li C, Ni D, Wu L, Zhu L, Wang H and Xu W. 2007. Molecular cloning, expression of a big defensin gene from bay scallop Argopecten irradians and the antimicrobial activity of its recombinant protein. Mol Immunol 44, 360-368.

Zhao J, Li C, Chen A, Li L, Su X and Li T. 2010. Molecular characterization of a novel big defensin from clam Venerupis philippinarum. PLoS ONE 5, e13480. 